\title{
Research on Monitoring and Control for Supply Chain Finance Risk
}

\author{
Wei Xing \\ Hunan Vocational College of Modern Logistics, Changsha, 410131, China \\ 760933676@QQ.com
}

Keywords: supply chain finance risk; monitoring process; control strategies; risk management theory

\begin{abstract}
Financial market is the artery of the whole market economy system. The rapidly development of supply chain finance has caused the supply chain financial risk. In this paper, the theory of supply chain financial risk management as a guide, the analysis of the supply chain financial risk monitoring process, put forward the supply chain financial risk control strategy. Specifically, it includes: excavating the value of financial services in the supply chain, avoiding systemic financial risks, strengthening the access management of logistics regulators, training high-quality supply chain financial operations teams, improving the supply chain financial risk control system and accelerating the development of electronic information platform Establish and perfect the legal system of supply chain finance. The research results of this paper play an important role in alleviating the financing difficulty of Small and Medium Enterprises, expanding the banking space and cultivating the competitiveness of the tertiary industry.
\end{abstract}

\section{Introduction}

With the deepening of socialized production mode, market competition has shifted from competition among single customers to competition between supply chain and supply chain. The supply chain for a given product, from raw material purchases to intermediate products and final products, is finally delivered by the sales network to consumers, connecting suppliers, manufacturers, distributors, retailers, and end-users altogether. In this supply chain, competitive and large-scale core enterprises, due to their strong position, are often harsh on the upstream and downstream supporting enterprises in terms of terms of trade such as delivery, price and account terms, thereby causing these enterprises A huge pressure. And most of the upstream and downstream supporting enterprises are Small and Medium Enterprises, it is difficult to finance from the bank, resulting in the final result of the capital chain is very tight, the entire supply chain imbalance. The biggest characteristic of "supply chain finance" is to find a big core enterprise in the supply chain and take the core enterprise as the starting point to provide financial support to the supply chain. On the one hand, the effective injection of funds into the relatively weak upper and lower reaches of supporting Small and Medium Enterprises to solve the problem of Small and Medium Enterprises financing difficulties and supply chain imbalance; the other hand, the bank credit into upstream and downstream business buying and selling behavior and enhance their commercial credit, promote Small and Medium Enterprises and core enterprises to establish long-term strategic synergies, enhance the competitiveness of the supply chain.

Finance is the core of the modern economy, and the financial market is the artery of the entire market economy. The high risk of the financial itself and the domino effect of the financial crisis make the safe, efficient and steady operation of the financial system crucial to the overall economic stability and development. The rapid development of supply chain finance has caused the supply chain financial risk. Supply chain financial risk refers to commercial banks and third-party logistics companies in the process of financing supply chain enterprises, due to a variety of unpredictable uncertainties in advance the impact of the actual supply chain financial products and expectations Deviations in earnings, or the likelihood that assets will not be recovered and thus suffered losses. Supply chain financial risk has a strong complexity and concealment, has become a key problem restricting the development of supply chain finance. This article studies the control and monitoring 
of complex supply chain financial risks, and provides services to alleviate the financing difficulties of Small and Medium Enterprises, expand the banking space and cultivate the competitiveness of the tertiary industry.

\section{Management Theory on Supply Chain Finance Risk}

Supply chain financial risk management includes the following basic theories [1,2]:

(1) Supply chain management theory. Through value-added processes and distribution channel controls, from supplier to customer, starting at the point of supply, ending at the end of consumption, emphasizing the complete chain from demand to supply. There are core enterprises in the supply chain, which are expanded from core enterprises to the supply chain before and after to form an integrated network, and the resources of the nodes in each network all flow in the network. Each node in the supply chain is a part of the whole supply chain, Not a simple combination, is the strategic integration and interdependence of the overall; integrated management of resources is the key to supply chain management; supply chain management depends on the integration and application of information technology.

(2) Asymmetric information theory. In the market economy activities, all kinds of personnel have different understanding of the relevant information. Those who have more information have a better position, while those who are poorly information are in a more disadvantaged position. The theory is that in the market, the seller knows more about the various kinds of information about the product than the buyer; the one with more information can benefit from the market by transmitting reliable information to the poor one; the less information is available from both the buyer and the seller One side, will strive to obtain information from the other party; market signals to a certain extent, make up for the problem of asymmetric information.

(3) Principal-agent theory. Principal-agent theory is one of the main contents of the contractual theory of institutional economics, which is mainly used to solve the problem of information asymmetry between the principal and the agent in the principal-agent relationship. For the principal, only to maximize the effectiveness of the agent's action in order to obtain the maximum benefits of its own effectiveness, which must be effective agents to stimulate. In the supply chain financial business relationship, there are principal-agent relations between financial institutions and the financing enterprises, logistics enterprises, raw material suppliers and downstream distributors in the supply chain and the nodes in the supply chain.

(4) Comprehensive risk management theory. In all aspects of the implementation of the basic risk management processes, cultivating a risk management culture, the establishment of a comprehensive risk management system, including risk management strategies, risk management measures, risk management organizational functions, risk management information systems and internal control systems for risk management The overall objective of providing assurance process and methods. The basic process of overall risk management: Establish the initial network of risk management information collection, carry out risk assessment, formulate risk management strategies, and propose and implement risk management solutions and risk management supervision and improvement.

\section{Monitoring Process on Supply Chain Finance Risk}

Any risk has a process of occurrence and development. The risk management process must be monitored in order to dynamically grasp the risks and their changes, and to track and control the risk management plan. Risk monitoring is through the entire process of risk planning, identification, analysis, assessment and response monitoring and control to ensure that risk management to achieve the desired goals. Risk monitoring activities are mainly to monitor the risk status, check the effectiveness of risk coping strategies, risk control activities are normal, the monitoring mechanism is perfect, constantly identify new risks, timely warning signals and the development of necessary countermeasures. Supply chain financial risk monitoring process shown in Fig. 1. 




Fig. 1. Monitoring process on supply chain finance risk

The input of the process of risk monitoring is the result of early risk identification, analysis, assessment and response activities such as risk management planning, risk response plan, risk control strategy, etc. By using continuous monitoring, individual evaluation or a combination of the two, With a variety of risk monitoring methods, techniques and tools, through the project resources, project risk control process control requirements, access to risk control results. In the supply chain financial risk monitoring process, you need to pay attention to the following questions:

(1) Systematic monitoring methods. First, the monitoring runs through the entire process of supply chain financial management, involving all stages of supply chain finance and various knowledge areas; second, the monitoring must be full participation, including financial institutions, Small and Medium Enterprises and core enterprises; Third, the monitoring has a hierarchy Sexual and top-level managers emphasize overall control and regulation, while bottom-level staff emphasize the details and norms of financial services. Fourthly, the monitoring system is of a holistic nature and must not separate financial operations or risks.

(2) Risk early warning system. In the process of supply chain finance, there are risks that may occur. By adopting advanced or precautionary management methods, once the risk signs are found in the monitoring process, timely corrective actions and early warning signals are issued in order to minimize the adverse consequences. The key of risk monitoring lies in cultivating acute risk awareness, establishing a scientific risk early warning system, changing from "fire fighting" to "fire fighting" risk monitoring, and from focusing on risk prevention to risk prior control.

(3) Risk contingency plans. Contingency planning is the preparation for a particular situation that may occur in the supply chain finance process, often caused by a risk "trigger." The triggers provide three basic control functions: one is to activate and provide alarm bell to revisit the risk action plan, the other is to release the signal to terminate the risk response activity and the third is to suspend the execution of the risk action plan. Including regular event triggers, relative change triggers and threshold triggers.

(4) Risk monitoring opportunity. Risk monitoring depends not only on the objective law of risk awareness, it is necessary to avoid risks, but also economically feasible. The risk management 
process must be cost-effective and try to find a balance between risk response and risk acceptance. The cost of risk control is estimated on the basis of the loss incurred in the absence of risk control and as the risk increases the loss increases. As risk control costs increase, the risk decreases. Therefore, to choose the appropriate timing of risk monitoring.

\section{Control Strategies on Supply Chain Finance Risk}

Different from the traditional credit business, the supply chain finance business has such characteristics as diversification of participants, complexity of business operation procedures and instability of the value of pledges, which determines that the risk control of supply chain financial business is different from the traditional credit business. Risk control refers to adopting various possible methods and measures to reduce the possibility of a risk event or reduce the value of risk loss when a risk occurs. The main methods of risk control are risk aversion, risk transfer, risk hedging and risk retention. Risk control is carried out on the basis of risk management planning. Once the risk is monitored, reasonable measures are taken to avoid the risk. Various measures can be taken to change the nature of the risk, change the probability of the risk happening, and change the impact of the risk. With reference to relevant literature [3-6], summarized the control strategy of supply chain financial risk as follows:

(1) Excavating the value of supply chain financial services. The significance of supply chain finance lies in the fact that financial institutions provide financial services only to a certain enterprise in the industrial chain and extend it to provide financial services to multiple enterprises in the industrial chain or the whole industry to maximize the value of financial services in the supply chain, Strengthen the supply chain financial risk prevention. Various enterprises in the supply chain are interdependent and frequent business activities are carried out between upstream and downstream enterprises. According to the laws of business operation, financial institutions summarize common needs and formulate standardized and comprehensive financial service solutions. With the real business context in the supply chain or credit enhancement conditions such as expected receivables, it is possible to provide financial services to Small and Medium Enterprises, thereby enhancing the quality of small and medium-sized customers.

(2) Avoid falling into the systemic financial risk. In the course of the development of supply chain finance, the characteristics of the financing chain around the development of the trade chain are very obvious. According to the correlation between the upstream and downstream enterprises in production and operation, they should rationally design products and provide financial services to related enterprises. Financial institutions must pay attention to the risks of the industry. Because the financial data of a single enterprise is not enough to reflect the trend of the whole industry, the trend of the market should be judged according to the national, industrial and regional policies, the overall grasp of the industry development trend, the full recognition of various potential risks in the industry, Credit policy to avoid falling into systemic risk. Financial institutions should, in cooperation with many enterprises in the supply chain, collect more industry data and make effective use of big data to conduct comprehensive and timely industry risk analysis.

(3) To strengthen the access management of the logistics regulatory party. In supply chain finance, logistics regulators play the role of supervisor, intermediary and information center. They not only professional management of customers' collateral, but also keep track of the dynamic changes of stocks in supply chain enterprises. In order to prevent non-standard operation of regulatory regulators and management system defects to financial institutions, should focus on the selection of large-scale operation, high visibility, good credit, storage equipment professional, advanced management technology, operational practices, strict monitoring procedures and high-quality staff Regulators to cooperate. Establish an irregular inspection system for regulators, intensify inspections, and supervise and improve regulators that do not meet regulatory requirements, with a firm exit if necessary.

(4) Cultivate high-quality supply chain financial operations team. Most of the illegal operations personnel belong to the internal crime and collusion both inside and outside, supply chain finance business mode of operation, making the internal staff and supply chain enterprises collusion crime 
increases the possibility. When selecting and cultivating supply chain financial business operators, we first select those business leaders who are experienced in the market and are familiar with the operation of the supply chain finance business. At the same time, we must regularly train and continuously improve our business capabilities, especially the risk control of supply chain financial businesses ability. Financial institutions through the training of operators on professional ethics and operational capabilities, to improve the quality of supply chain financial operations staff and reduce the loss of operational risk due to personnel factors.

(5) Improve supply chain financial risk control system. Before lending, financial institutions earnestly assess the risks of credit enterprises, measure the causes of risks and risk coefficients comprehensively, strive to improve self-control from the source and identify risks, and provide a better internal control environment for supply chain finance. During the operation of supply chain finance, the bank is in a passive position to strengthen risk monitoring in loans and effectively guarantee the safety of credit funds. In the process of supply chain finance, the risk is contagious. Financial institutions must pay attention to risk early warning, establish early-warning mechanism for post-loan risk, improve the accuracy and systematic of risk search, and help financial institutions carry out scientific risk management to prevent risks The occurrence and proliferation.

(6) Accelerate the construction of electronic information platform. In supply chain finance mode, the information flow existing in the supply chain can make it easier for financial institutions to obtain the relevant information of credit enterprise and control the financing risk to the maximum extent. Through the establishment of an electronic information platform, we can realize the information management of total supply chain financial business, business structure, financing products and supervised enterprises, so as to realize the day-to-day financing pledge and detention operations, reporting statistics, risk warning information, inventory and redemption Goods and other electronic work, so that business processes flow and transparency, reduce reliance on personnel, reduce artificial arbitrary. Electronic information platform operation guide function, error correction function and risk warning function, can reduce the operator's error probability.

(7) Improve the legal system of supply chain finance. Law is a laggard reflection of the law of social and economic life, but orderly economic activity needs a sound legal system as a support. Supply chain finance as a new business, the development time is short, the relevant laws need to go through the imperfections to improve the process. The state legislature should improve relevant laws on supply chain finance as soon as possible to further improve the system for quarantine of movable property and chattel mortgage. The supply chain finance business involves multiple parties and the ownership of pledges also flows among the entities. Various contracts are required to maintain Equal and orderly cooperation among the main bodies provides mandatory rules for supply chain management and accelerates the process of economic and financial reform.

\section{References}

[1] X. H. Li, "On the Risk Control of Bank A's Supply Chain Finance," Master's Dissertation of Chongqing University of Technology, 2015.

[2] S. H. Li, "Research on Warning and Controlling of Supply Chain Financial Risks," Doctor's Degree of Wuhan University of Technology, 2014.

[3] Y. Yu, Y. Q. Yang, "Discussion of Supply Chain Finance Risk Management," Logistics Sci-Tech, vol. 38, no. 4, pp. 86-88, 2014.

[4] C. Fei, "Financial risk control in supply chain," Logistics Technology, vol. 23, no. 2, pp. 42-43, 2016.

[5] G. Y. Zhu, "Risk Control for Supply Chain Financing Businesses of TPL Enterprises," Logistics Technology, vol. 33, no. 17, pp. 329-331 2015.

[6] C. Y. Kang, "The Research on Supply Chain Finance Mode and Risk Control," Journal of Changchun Finance College, vol. 32, no. 5, pp. 25-31, 2017. 